\title{
BNV/LNV Searches in Charmonium Decays at BESIII
}

\section{Minggang Zhao*}

(On behalf of the BESIII Collaboration)

School of Physics, Nankai University,

94 Weijin Rd, Nankai District, Tianjin, China

E-mail: zhaomg@nankai.edu.cn

The observed matter-antimatter asymmetry in the universe composes a serious challenge to our understanding of nature. BNV/LNV decay has been searched in many experiments to understand this large-scale observed fact. In the case of $e^{+} e^{-}$collision, few experiments are performed. Here we proposed to search BNV and LNV with currently the world largest $\mathrm{J} / \mathrm{psi}$ data sets in e+e- collision experiment. The BNV and LNV channel $J / \psi \rightarrow \Lambda_{c}^{+} e^{-}+$c.c. is searched, and no signal event is observed. The upper limit of the branching fraction is set to be $6.9 \times 10^{-8}$ at $90 \%$ Confidence Level, which is still much higher than the estimation based on SM. For the process with $\Delta B=2$, a search for $\Lambda-\bar{\Lambda}$ oscillation is performed for the first time. No evidence of the baryon oscillation is observed, the upper limit for the rate of $\bar{\Lambda}$ oscillates to $\Lambda$ is determined as $\mathcal{P}(\Lambda)=\frac{\mathcal{B}\left(J / \psi \rightarrow p K^{-} \Lambda+c . c .\right)}{\mathcal{B}\left(J / \psi \rightarrow p K^{-} \bar{\Lambda}+c . c .\right)}<4.4 \times 10^{-6}$, and the oscillation parameter $\delta m_{\Lambda \bar{\Lambda}}$ is calculated to be less than $3.8 \times 10^{-15} \mathrm{MeV}$ at $90 \%$ confidence level.

40th International Conference on High Energy physics - ICHEP2020

July 28 - August 6, 2020

Prague, Czech Republic (virtual meeting)

${ }^{*}$ Speaker 


\section{Introduction}

The asymmetry of matter and anti-matter is a big problem in the evolution of universe from the Big Bang to the matter dominated state. Three rules are proposed by Sakharov[1] to understand the problem: Charge $(C)$ and Charge-Parity $(C P)$ violation, Baryon Number violation (BNV), and existence of thermal nonequilibrium. $C$ and $C P$ violation predicted in the scope of the Standard Model were precisely tested by many experiments. However, BNV has not been observed by any experiments.

The BNV would imply the instability of the proton and the nucleus, albeit at a time scale of the order of the lifetime of the proton [2]. There are many theoretical models in which baryon number is not an exact symmetry of the Nature. For example, in the Grand Unified Theory (GUT), proton can decay into several mode through leptoquarks [3] such as $p \rightarrow e^{+} \pi^{0}$ etc. This mechanism simultaneously breaks the baryon number $(\mathrm{B})$ and the lepton number $(\mathrm{L})$ conservation while keeping constant the difference (B-L). Although the negative results of proton decay experiments almost rule out the parameter space of the simplest $B-L$ GUTs, this is definitely not the end of story for searching grand unifications which allow the BNV. For example, the $\mathrm{SO}(10)$ model, E6 model and flipped SU(5) model, all predict a longer proton lifetime which does not conflict against the data. Since the understanding of the GUT and the universe evolution are a long-time pursuit of physicists, searching for BNV experimentally will never cease.

On the other hand, as discussed in Ref. [4], recent discoveries of neutrino oscillations have made $N-\bar{N}$ oscillation to be quite plausible theoretically if small neutrino masses are to be understood as a consequence of the seesaw mechanism [5], which indicates the existence of $\Delta(B-L)=2$ interactions. There has been many experimental searches [6] for neutron-antineutron oscillation since 1980 [7], while few results from other baryons. In 2010, it was pointed out by Ref. [8] that the $\Lambda-\bar{\Lambda}$ oscillation can be investigated at the BESIII experiment. And there are no experimental searches till now. One more advantage is the fact that $\Lambda$ contains a second-generation quark, while proton and neutron consist of the first generation quarks only. That is why the search of $\Lambda-\bar{\Lambda}$ oscillation allows extending the BNV studies in proton decay and neutron-antineutron oscillation experiments.

As being well known, the two independent ways of searching for new physics at collider and non-collider experiments are fruitfully supporting each others, moreover they might also find and correct errors of either side. So searching for BNV process at collider experiments might provide some different information compared with the proton decay experiments.

\section{Search for Baryon and Lepton Number Violation in $J / \psi \rightarrow \Lambda_{c}^{+} e^{-}+$c.c.}

Based on the $1.31 \times 10^{9} \mathrm{~J} / \psi$ events collected by the BESIII detector at the Beijing Electron Positron Collider, we search for the process $J / \psi \rightarrow \Lambda_{c}^{+} e^{-}+$c.c. for the first time, where the $\Lambda_{c}^{+}$ is reconstructed through the decay $\Lambda_{c}^{+} \rightarrow p K^{-} \pi^{+}$. In this process, both baryon and lepton number conservation is violated. The candidate events of $J / \psi \rightarrow \Lambda_{c}^{+} e^{-}$are studied by examining the invariant mass of the $p K^{-} \pi^{+}$system, $M_{p K^{-} \pi^{+}}$, as shown in Fig. 1 .

Since no events are observed in the signal window, the upper limit on the branching fraction $\mathcal{B}\left(J / \psi \rightarrow \Lambda_{c}^{+} e^{-}+\right.$c.c. $)$is set to be $6.9 \times 10^{-8}$ at the $90 \%$ Confidence Level, which is more than two 


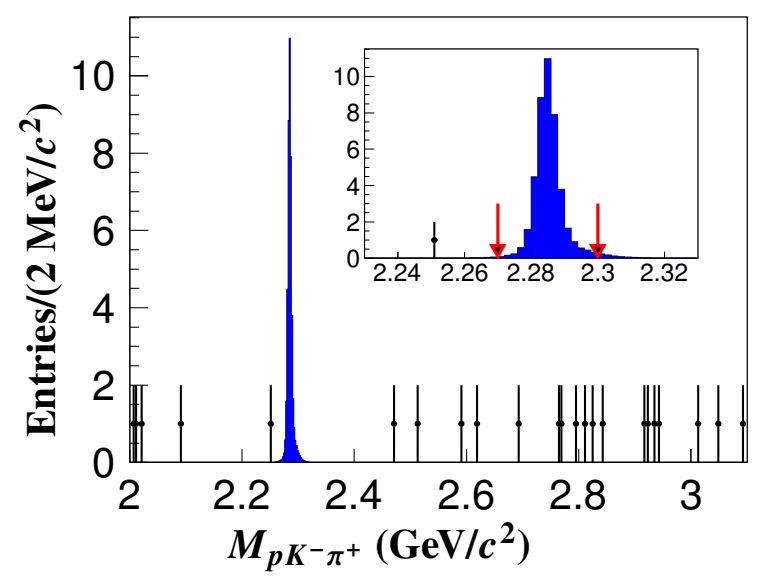

Figure 1: Distributions of $M_{p K^{-} \pi^{+}}$for the $J / \psi \rightarrow \Lambda_{c}^{+} e^{-}$candidate events for signal MC simulation (shaded histogram) and data (dots with error bars), where the signal MC sample is normalized arbitrarily. The inset plot shows a narrow mass range within $(2.23,2.33) \mathrm{GeV} / c^{2}$, where the arrows represent the signal mass window.

orders of magnitude more strict than that of CLEO's measurement in the analogous process [9]. The result is one of the best constraints from meson decays $[10,11]$ and is consistent with the conclusion drawn from the proton decay experiment [12].

\section{Search for $\Lambda-\bar{\Lambda}$ oscillation in $J / \psi \rightarrow p K^{-} \bar{\Lambda}$}

By analyzing the $1.31 \times 10^{9} \mathrm{~J} / \psi$ events, a search for $\Lambda-\bar{\Lambda}$ oscillation is performed for the first time based on the decay $J / \psi \rightarrow p K^{-} \bar{\Lambda}$ with the $\bar{\Lambda}$ possibly oscillates to $\Lambda$, as shown in Figure 2. Such a decay violates baryon number conservation with $\Delta B=2$.
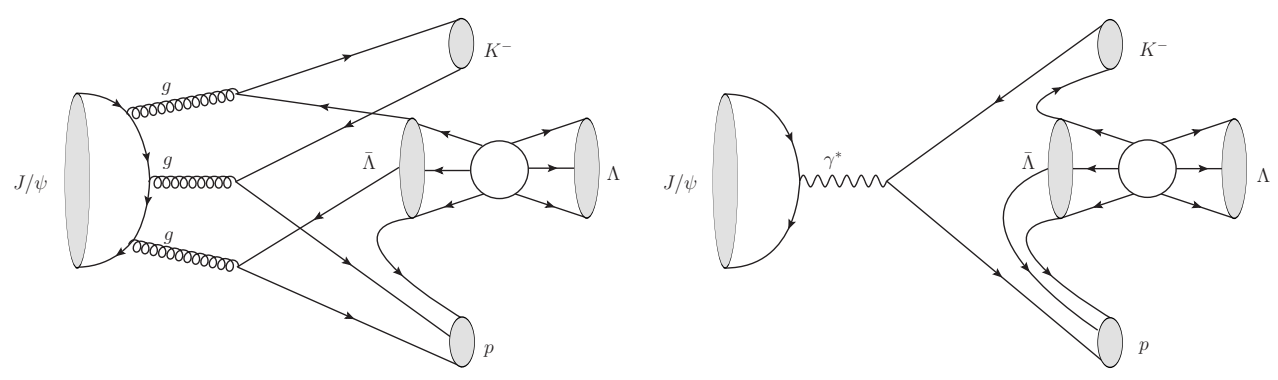

Figure 2: Illustration of $\Lambda-\bar{\Lambda}$ oscillation from strong (left) and electro-magnetic (right) $J / \psi$ decays.

The concerned decay channels are $J / \psi \rightarrow p K^{-} \bar{\Lambda}$ and $J / \psi \rightarrow p K^{-} \Lambda$, where $\Lambda(\bar{\Lambda})$ is reconstructed by its decay to $p \pi^{-}\left(\bar{p} \pi^{+}\right)$. Therefore the particles in the final state are $p \bar{p} K^{-} \pi^{+}$or $p p K^{-} \pi^{-}$. We define the events from the decay $J / \psi \rightarrow p K^{-} \bar{\Lambda}$ as Right Sign (RS) events, while the ones from $J / \psi \rightarrow p K^{-} \bar{\Lambda} \rightarrow p K^{-} \Lambda$ as Wrong Sign (WS) events. The candidate signal events are studied by examining the invariant mass spectra of $p \pi$ final state, $M_{p \pi}$ as shown in Figure 3 where the number 
of events for RS and WS are obtained. Since there is no events survived within the signal region, no evidence of baryon anti-baryon oscillation is observed, the upper limit for the rate of $\bar{\Lambda}$ oscillates to $\Lambda$ is determined as

$$
\mathcal{P}(\Lambda)=\frac{\mathcal{B}\left(J / \psi \rightarrow p K^{-} \Lambda \rightarrow p K^{-} p \pi^{-}\right)}{\mathcal{B}\left(J / \psi \rightarrow p K^{-} \bar{\Lambda} \rightarrow p K^{-} \bar{p} \pi^{+}\right)}=\frac{N_{\mathrm{WS}}^{\mathrm{obs}} / \epsilon_{\mathrm{WS}}}{N_{\mathrm{RS}}^{\mathrm{obs}} / \epsilon_{\mathrm{RS}}}<4.4 \times 10^{-6},
$$

and the oscillation parameter $\delta m_{\Lambda \bar{\Lambda}}$ is measured to be

$$
\delta m_{\Lambda \bar{\Lambda}}<3.8 \times 10^{-15} \mathrm{MeV}
$$

at $90 \%$ confidence level.

Our result is comparable with the prospective onstraint in Ref. [8] with only about one-tenth data sample. This is the first measurement on $\Lambda-\bar{\Lambda}$ oscillation which is an alternative way to search for $\mathrm{BNV}$ process with $\Delta B=2$ in addition to neutron oscillation experiments.

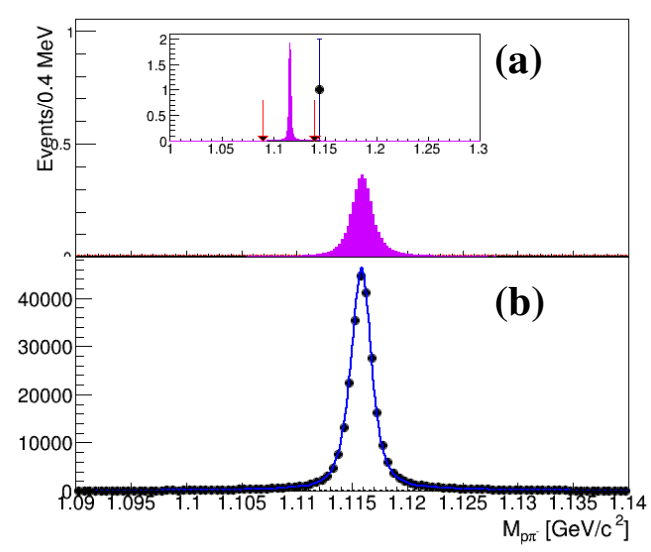

Figure 3: The $M_{p \pi}$ distribution of (a) WS events in the signal region and full span, where the dot with error bar is from data, the pink filled histogram which is normalized arbitrarily is from simulated WS signal events, the arrows in the inset figure show the edges of signal region, (b) RS events from data, where the dots with error bars are from data and the blue line represents the fitting result in which the signal shape is modeled with MC simulated shape convoluted with a Gaussian function and the background shape is obtained from inclusive MC sample after excluding RS events.

\section{Summary}

The results of BNV searching at BESIII experiment are consistent with the complementary/compatible experiments. Since BNV plays key role to reveal the nature of revolution of the universe, never too much efforts are expected. For example, with 10 billion new $J / \psi$ data collected recently with the BESIII detector, the expected constraints on $\mathcal{B}\left(J / \psi \rightarrow \Lambda_{c}^{+} e^{-}\right)$and $\delta m_{\Lambda \bar{\Lambda}}$ can be improved with about one order or even better. And in the proposed next generation super $\tau$-charm factory, several trillion $J / \psi$ events might be possible, then the constraints will be much better. 


\section{Acknowledgement}

The BESIII collaboration thanks the staff of BEPCII and the IHEP computing center for their strong support. The speaker would thank the NSFC under Contracts Nos. 12035009,11875170 and the Joint Large-Scale Scientific Facility Funds of the NSFC and CAS under Contracts Nos. U1832207 and U1932102. The talk/work is supported by them.

\section{References}

[1] A. D. Sakharov, JETP Lett. 5, 24 (1967).

[2] F.C. Adams and G. Laughlin, Rev. Mod. Phys. 69, 337 (1997).

[3] J. C. Pati and A. Salam, Phys. Rev. D 10, 275 (1974); Phys. Rev. D 11, 703 (1975).

[4] B. Dutta, Y. Mimura, and R.N. Mohapatra, Phys. Rev. Lett. 96, 061801 (2006).

[5] P. Minkowski, Phys. Lett. B 67, 421 (1977); R.N. Mohapatra and G. Senjanovic, Phys. Rev. Lett. 44, 912 (1980).

[6] P. A. Zyla et al. (Particle Data Group), Prog. Theor. Exp. Phys. 2020, 083 C01 (2020).

[7] R. N. Mohapatra and R.E. Marshak, Phys. Rev. Lett. 44, 1316 (1980)

[8] X.-W. Kang, H.-B. Li and G.-R. Lu, Phys. Rev. D 81 051901(R) (2010).

[9] P. Rubin et al. (CLEO Collaboration), Phys. Rev. D 79, 097101 (2009).

[10] M.E. McCracken et al. (CLAS Collaboration), Phys. Rev. D 92, 072002 (2015).

[11] P. del Amo Sanchez et al. (BABAR Collaboration), Phys. Rev. D 83, 091101(R) (2011).

[12] K. Abe et al. (Super-Kamiokande Collaboration), Phys. Rev. D 95, 012004 (2017); K. Abe et al. (Super-Kamiokande Collaboration), Phys. Rev. D 96, 012003 (2017). 